\title{
Use of Quantitative Real-Time PCR to Unravel Ecological Complexity in a Biological Control System
}

\author{
Guy R. Knudsen1, Tae Gwan Kim², Yeoung-Seuk Bae³, Louise-Marie C. Dandurand4 \\ ${ }^{1}$ Soil \& Land Resources Division, University of Idaho, Moscow, Idaho, USA \\ ${ }^{2}$ Department of Environmental Science and Engineering, Ewha Womans University, Seoul, Republic of Korea \\ ${ }^{3}$ International Technology Co-Operation Center, Rural Development Administration, Suwon, Republic of Korea \\ ${ }^{4}$ Plant Science Division, University of Idaho, Moscow, Idaho, USA \\ Email: gknudsen@uidaho.edu
}

Received 16 March 2015; accepted 31 March 2015; published 2 April 2015

Copyright (C) 2015 by authors and Scientific Research Publishing Inc.

This work is licensed under the Creative Commons Attribution International License (CC BY).

http://creativecommons.org/licenses/by/4.0/

(c) (i) Open Access

\begin{abstract}
Biological control of soilborne plant pathogens using beneficial fungi, such as the mycoparasite Trichoderma harzianum, offers the prospect of environmentally benign pest control. However, biocontrol organisms have their own natural enemies; for example the fungivorous nematode Aphelenchoides saprophilus preys on T. harzianum. A trophic cascade occurs when three or more trophic levels are present in a food chain, and consumption of the intermediate species affects biomass or productivity of a lower trophic level; such an interaction in this system might reduce the biocontrol efficacy of $T$. harzianum. However, the presence of refuges, where intermediatelevel species are protected from predation, may reduce the ecological impact of a trophic cascade. Interactions among microscopic organisms in a complex medium such as soil are difficult to observe and quantify. We evaluated the potential of quantitative real-time PCR (qRT-PCR) as a tool to investigate the trophic cascade interaction among $T$. harzianum, $A$. saprophilus, and the plant pathogen Sclerotinia sclerotiorum. Results indicate that the mycoparasite colonized and persisted inside structures (sclerotia) of the target plant pathogen, where it was relatively protected from predation compared to the surrounding soil environment. In this way, colonization of sclerotia may provide a refuge that reduces trophic cascade effects in this system. qRT-PCR provided a sensitive method to investigate fungal dynamics over time in this multitrophic system.
\end{abstract}

\section{Keywords}

qRT-PCR, Biocontrol, Nematode, Predation, Trophic Cascade

How to cite this paper: Knudsen, G.R., Kim, T.G., Bae, Y.-S. and Dandurand, L.-M.C. (2015) Use of Quantitative Real-Time PCR to Unravel Ecological Complexity in a Biological Control System. Advances in Bioscience and Biotechnology, 6, $237-244$. http://dx.doi.org/10.4236/abb.2015.64023 


\section{Introduction}

Reducing chemical pesticide use is a desirable goal for agriculture and forestry. One disease control method, the application of fungi or bacteria as microbial antagonists of plant pathogens, offers prospects of environmentally benign pest control. Biological control (biocontrol) microbes applied to seeds or soil may colonize soil or the rhizosphere, and thus may be present at or near infection courts of soilborne pathogens. There, biocontrol agents may reduce disease through a variety of mechanisms including production of antifungal compounds, hyperparasitism of pathogens, stimulation of host plant defenses, or competitive colonization of the spermosphere or rhizosphere. Numerous fungi have shown potential as agents for biological control of soilborne phytopathogens. However, soils are densely populated and highly competitive habitats, and poor competitive ability of introduced fungi, leading to unsuccessful establishment and activity, has often been observed [1]. Unlike gnotobiotic systems, soil is characterized by a multitude of both trophic (i.e., food web) and non-trophic (e.g., mutualism, commensalism, amensalism, antagonism, competition) interspecific relationships [2]. Thus, ecological complexity within the soil biotic community may significantly affect the success of biological control of plant pathogens using natural enemies.

The genus Trichoderma contains several species, especially $T$. harzianum, that have been extensively evaluated as biocontrol agents against soilborne fungal pathogens [3] [4], including the sclerotium-forming fungus Sclerotinia sclerotiorum, where as a mycoparasite T. harzianum can colonize and kill the resting stage of this pathogen [5]. However, we have also demonstrated that the fungivorous nematode Aphelenchoides saprophilus is able to feed on hyphae of $T$. harzianum in soil [6] (Figure 1).

Where three or more trophic levels are present in a food chain, consumption of prey by top predators can induce cascading effects (known as "trophic cascades") on the abundance, biomass or productivity on organisms of lower trophic levels [7]. Trophic cascades occur when predators in a food web suppress the abundance of their prey, releasing the next lower level from predation by the intermediate level [7]. We hypothesized that the Aphelenchoides $\times$ Trichoderma $\times$ Sclerotinia food chain represents such a trophic cascade, in that fungivory by A. saprophilus reduces the growth of $T$. harzianum and its ability to successfully colonize sclerotia of $S$. sclerotiniorum, effectively reducing mycoparasitism (and thus reducing biocontrol) of the plant pathogen.

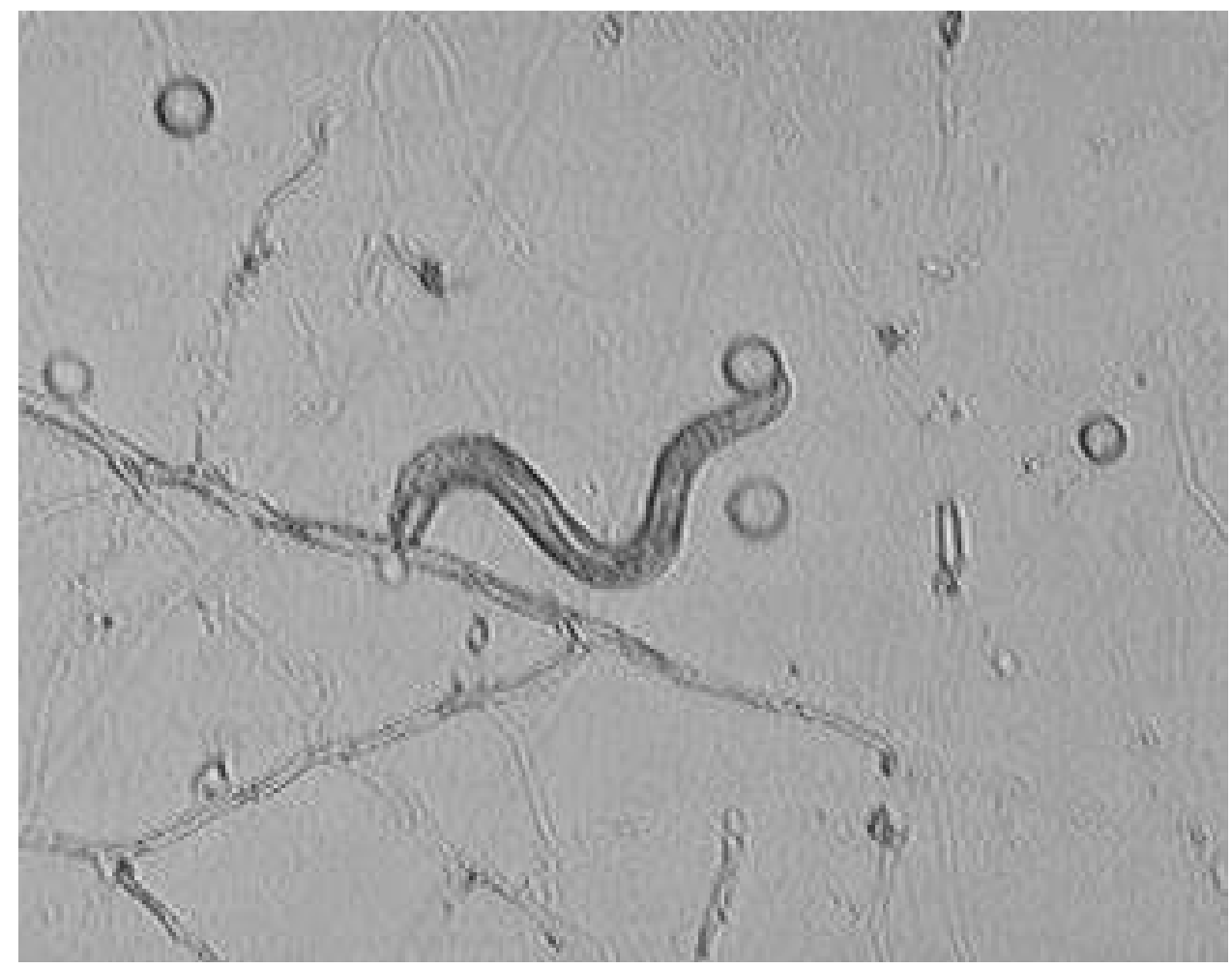

Figure 1. Aphelenchoides saprophilus feeding on a hypha of Trichoderma harzianum. 
In addition, it has been suggested that the presence of refuges for intermediate species in the trophic chain can act to stabilize cascades [8]. We hypothesized that once hyphae of $T$. harzianum penetrate and colonize sclerotia of $S$. sclerotiorum, effects of fungivorous nematodes may be lessened, since their ability to access $T$. harzianum may be reduced compared to in soil. We hypothesized that in this way, colonized sclerotia potentially represent a refuge where the biocontrol agent is protected from predation by nematodes, thereby reducing the trophic cascade effect. However, for soil microbes, assessment of trophic interactions is especially challenging due to difficulties in identifying and quantifying microbial species in soil. In this study, we evaluated the potential of quantitative real-time PCR (qRT-PCR) as a tool to investigate a trophic cascade interaction among three species (S. sclerotiorum, T. harzianum, A. saprophilus) in a soil food web, and the ability of $T$. harzianum to utilize colonized sclerotia as a refuge from predation by the fungivorous nematode.

\section{Materials and Methods}

\subsection{Organisms Used}

Trichoderma harzianum ThzID1-M3 is a transformant strain of Thz (wild type) ThzID1. The parental isolate was obtained from field soil near Moscow, Idaho.ThzID1-M3 was derived by PEG-mediated cotransformation of ThzID1 with genes encoding GFP and hygromycin B resistance; it is mitotically stable, similar in morphology and biocontrol activity to the parent, and expression of GFP in soil has been demonstrated [9]. ThzID1-M3 was formulated with alginate as previously described [5]: the fungus was allowed to grow for 1 week on potato dextrose agar at 21C, and three 1- $\mathrm{cm}^{2}$ pieces from sporulating culture were transferred to a 1-liter flask containing $500 \mathrm{ml}$ potato dextrose broth. Flasks were placed on a rotary shaker $(120 \mathrm{rpm})$ at room temperature and incubated for 1 week with 12 hours of light per day. Cultures were strained through cheesecloth and rinsed thoroughly with sterile water. Thirty-seven grams of hyphal biomass was blended briefly with 2 grams of wheat bran and $100 \mathrm{ml}$ of $1 \%$ aqueous sodium alginate solution. Drops of the mixture were added to $0.25 \mathrm{M} \mathrm{CaCl}_{2}$ solution, forming a pellet precipitate. Pellets were removed by straining, placed on waxed paper to air-dry at room temperature for 2 days then stored at 4C prior to use. The fungivorous nematode Aphelenchoides saprophilus was obtained from field soil near Moscow, ID, and populations were maintained on agar cultures of $T$. harzianum [6]. An isolate of S. sclerotiorum was grown on PDA for 7 days, then transferred to carrot discs and incubated 6 - 8 weeks at 25 8C. Sclerotia were harvested and airdried, then preconditioned for carpogenic germinationby incubating them on moist (saturated) sand at $10 \mathrm{C}$ in the dark for approximately 24 months.

\subsection{Soil}

Latahco silt loam soil was obtained from the University of Idaho plant science farm near Moscow, ID. Soil analysis results (University of Idaho Analytical Services Laboratory) indicated that the soil contained $20 \%$ sand, $20 \%$ clay and $60 \%$ silt by weight, with $82.2 \mu \mathrm{g} / \mathrm{g}$ of plant-available iron per gram. Soil $\mathrm{pH}$ in soil/water (2:1) solution was approximately 5.9. Soil was sieved through a $2 \mathrm{~mm}$ mesh and allowed to air-dry at room temperature prior to use.

\subsection{PCR Primers and Probes}

Trichoderma harzianum ThzID1, ThzID1-M3, and S. sclerotiorum were allowed to grow in 1-Liter flasks containing $500 \mathrm{ml}$ of potato dextrose broth for $4 \mathrm{~d}$ at room temperature on a rotary shaker (120 rpm). Mycelium was harvested by filtering through cheesecloth and rinsing with sterile distilled water, then ground in a porcelain mortar with liquidnitrogen. Total genomic DNA was extracted using the DN easy plant DNA extraction kit (Qiagen, Valencia, CA, USA) according to the manufacturer0s instructions. Extracted DNA was eluted twice in $100 \mathrm{ml}$ of Buffer AE (Qiagen) and the elutions were combined. Purified DNA was quantified spectrophotometrically, and stored at $-20 \mathrm{C}$ prior to use. Several primer/probe sets specific for ThzID1-M3were designed based on a GFP gene sequence (Gen Bank Accessionno. U55761) using Beacon designer software versions 2 and 4 (Premier Biosoft International, Palo Alto, CA, USA). The Basic Local Alignment Search Tool (BLAST) was used to confirm the absence of significant homology with other known DNA sequences. Eight different primers and the probes exhibited low homology with sequences of soil microorganisms, including generic Trichoderma spp. For selected primer sets that did not give unintended signals (such as primer dimers and nonspecific bands), BLAST was repeated to find a probe with the lowest homology with other known fungal sequences. The best 
primer/probe set, designated TMeGFP2, contained a FAM reporter dye conjugated to the 50-terminaland a TAMRA quencher dye conjugated to the 30-terminal (Table 1 ).

To quantify S. sclerotiorum DNA, we used the TMSCL2 primer/probe set (Integrated DNA Technologies, Coralville, IA, USA) based on the S. sclerotiorum calmodulin gene sequence as described previously [10]. The TMSCL2 probe contained a HEX reporter dye andTMeGFP2 primer/probe set (Table 1). PCR conditions resulting in the lowest cycle threshold $(\mathrm{Ct})$ value and the highest normalised fluorescence were selected, and PCR annealing and extension temperatures were optimised at 58 - 63 C. Standard curves obtained from each of the TMeGFP2 and TMSCL2 sets generated a linear fit with a slope of 3.4 to 3.6 with coefficient of determination $\left(R^{2}\right)$ values greater than 0.99 , with a PCR efficiency higher than $90 \%$ over at least six orders of magnitude.

\section{4. qRT-PCR Standards}

PCR standards were developed as follows: first, purified DNA of T. harzianum ThzID1-M3 or S. sclerotiorum was serially diluted (1:10). Standard curves were constructed by plotting threshold cycles (Ct) for ten-fold dilution series of the extracted pure DNA vs. logarithmically transformed DNA amounts of the dilution series. Ct value specified as the cycle number at which the fluorescent signal exceeded background fluorescence, a linear regression equation of a standard curve, and PCR efficiency were determined by the Bio-Rad iCycler IQ software program version 3.1 (Bio-Rad, Hercules, CA). ThzID1-M3 DNA standards ranged from $170 \mathrm{fg}$ to $170 \mathrm{ng}$ and S. sclerotiorum DNA standards ranged from $1 \mathrm{pg}$ to $100 \mathrm{ng}$. Real-time PCR amplifications of total DNA extracted from sclerotia were performed using the iCycler IQ (Bio-Rad). The reactions were carried out in a realtime PCR plate (VWR International, West Chester, PA) with a total volume of $25 \mathrm{ml}$ per single reaction.

The TMeGFP2 set was tested for potential false positive reactions with DNA from soil by adjusting nonsterile soil to a moisture content of either $-50 \mathrm{kPa}$ or $-500 \mathrm{kPa}$, then added $20 \mathrm{~g}$ of soil to plastic containers $(8 \mathrm{~cm} \times 6.3$ $\mathrm{cm} \times 6.3 \mathrm{~cm}$ ) and incubating at $22 \mathrm{C}$. After $0,3,5,7,14$, and $21 \mathrm{~d}$, replicate containers were destructively sampled. Soil was homogenized by suspending in $80 \mathrm{ml}$ of $50 \mathrm{mM}$ sodium phosphate buffer [pH 7.0], and placing on a rotary shaker (200 rpm) for $1 \mathrm{~h}$. Total DNA was extracted from a $0.25 \mathrm{ml}$ subsample from each suspension, using the PowerSoil DNA isolation kit (Mo Bio Laboratories, Carlsbad, CA, USA). qRT-PCR amplification of extracted DNA was performed using the TMeGFP2 primer/probe set, with conditions as described above. No false positive results were obtained from soil that wasn't inoculated with T. harzianum ThzID1M3.

To correlate fungal biomass with qRT-PCR results, known weights (1, 2, 3, 4, 8 or 9 mg, dry weight) of mycelium were placed in $1.5 \mathrm{ml}$ microtubes and ground with liquid nitrogen. Total genomic DNA was extracted using the DNEasy plant DNA extraction kit (Quiagen Inc., Valencia, CA, USA). DNA was twice eluted in100 $\mathrm{ml}$ of AE buffer, and the elutions were combined and stored at $20 \mathrm{C}$ prior to use. The relationship between fungal biomass and DNA estimates, for all three species, using the qRT-PCR assay was quantified using linear regression. All standard curves obtained from qRT-PCR runs with the above primer/probe sets generated a linear fit with R2 $\geq 0.98$, with $\geq 90 \%$ PCR efficiencies.

\subsection{Trophic Cascade Interactions: Fungivory of T. harzianum by A, saprophilus, and Colonization of Sclerotia vs. Surrounding Soil by T. harzianum ThzID1-M3}

Ability of A. saprophilus to reduce both colony radial growth and recoverable populations of T. harzianum in

Table 1. TMeGFP2 and TMSCL2 primer/probe sets to quantify DNA of Trichoderma harzianum ThzID1-M3 and Sclerotinia sclerotiorum.

\begin{tabular}{crcc}
\hline Primer/probe & Sequence & Target organism & Position \\
\hline TMeGFP2F $^{\mathrm{a}}$ & 5'-GCTGCCCGACAACCACTAC-30 & T. harzianum ThzID1-M3 & 585 \\
TMeGFP2R $^{\mathrm{b}}$ & 5'-CGTCCATGCCGAGAGTGATC-30 & 706 \\
TMeGFP2P $^{\mathrm{c}}$ & 5'-FAM-CGGCGGCGGTCACGAACTCCA-TAMARA-30 & - $^{\mathrm{d}}$ & \\
TMSCL2F $^{\mathrm{a}}$ & 5'-CCCAGTTCGACTCTCCTCTTTTAT-30 & 148 \\
TMSCL2R $^{\mathrm{b}}$ & 5'-AACTCAGACTCGGAAGGGTTTTG-30 & 359 \\
TMSCL2P $^{\mathrm{c}}$ & 5'-HEX-AGACATCTTGACCGACACCGCCCC-IBFQ-30 & & 181 \\
\hline
\end{tabular}

${ }^{a}$ Forward primers. ${ }^{b}$ Reverse primers. ${ }^{\mathrm{c}}$ TaqMan probes: T. harzianum ThzID1-M3 probe is labelled with the reporter dye FAM (6-carboxy-fluorescein) on the 5' end and the quencher dye TAMRA (6-carboxy-tetramethyl-rhodamine); S. sclerotiorum probe is labelled with the reporter dye HEX (hexachlorofluorescein) and the quencher dye IBFQ (Iowa Black FQ by Integrated DNA Technologies). ${ }^{\mathrm{d}}$ Antisense probe. 
soil was previously shown [6]. Nematodes (10 nematodes/g of soil, males and females, mixed life stages) were added to soil with pelletized T. harzianum; controls consisted of sterile water. After 5, 10, 20, or 30 days, recoverable populations of ThzID1-M3 and total recoverable Trichoderma spp. were determined by plating samples on selective media, and nematodes were extracted with a Baermann funnel and counted.

We investigated the possibility that colonized sclerotia may serve as refuges that enhance survival of $T$. harzianum, compared to survival in the surrounding soil, as follows. T. harzianum ThzID1-M3 was formulated into pellets as described above. Sclerotia of S. sclerotiorum (surface-sterilized in $10 \%$ bleach solution) were attached to glass microscope slides using cyanoacrylate glue, either withThzID1-M3 pellets attached approximately 15 $\mathrm{mm}$ away from sclerotia, or without ThzID1-M3 pellets. The slides then were placed in Petri dishes which were filled with nonsterile soil, with nematodes present, adjusted to $-100 \mathrm{kPa}$ matric potential. The dishes were sealed with parafilm to maintain a relatively constant moisture content, and were incubated at $22 \mathrm{C}$. After 20, 30, or 40 $\mathrm{d}$, sclerotia were (destructively) sampled and either sectioned for microscopic observation or held at $-20 \mathrm{C}$ for subsequent DNA extraction and qRT-PCR analysis. Sclerotia sampled for microscopic observation were embeddedin paraffin, and $20 \mathrm{~mm}$ thick sections were cut with a microtome. Sections were viewed at $400 \times$ magnification using a Nikon Eclipse E1000 epifluorescence microscope (Nikon, Melville, NY, USA), to observe GFP fluorescence of ThzID1-M3. From captured images, fluorescing ThzID1-M3 hyphal segments were quantified using Image Pro Plusimaging software (Media Cybernetics, Bethesda, MD, USA). Hyphal segments were quantified based on pixel numbers on the images, and values then were converted to area $\left(\mathrm{mm}^{2}\right)$. A haemocytometer was used as a measuring grid to calibrate an appropriate conversion factor.

Amounts of T. harzianum ThzID1-M3 DNA as well as S. sclerotiorum DNA inside sclerotia were quantified using the real-time PCR assay with the TMeGFP2 and TMSCL2 primer/probe sets. Sclerotia were ground with liquid nitrogen, and DNA was extracted using the DN easy plant DNA kit. Amounts of S. sclerotiorum DNA and ThzID1-M3 DNA contained in each sclerotium were determined as described above.

Also, in order to quantify ability of T. harzianum ThzID1-M3 to grow and persist outside of sclerotia, at each sample time the surrounding soil was sampled and the amount of $T$. harzianum ThzID1-M3 DNA there was quantified. Total DNA was extracted from $0.25 \mathrm{ml}$ (containing $0.05 \mathrm{~g}$ soil) of each homogenized soil suspension using the Power Soil DNA Isolation kit.

We also used epifluorescence microscopy of the GFP-expressing ThzID1-M3 to quantify hyphal proliferation (biomass) in the same treatments, using the methods of Orr and Knudsen [11]. Subsamples (0.01 g of soil) from each homogenized soil suspension were filtered through $0.8-\mu \mathrm{m}$ membrane filters and viewed at $100 \times$ with a Nikon Eclipse E1000 epifluorescence microscope (Nikon, Melville, NY) to observe hyphal fragments with GFP activity. Membrane filters were prepared and examined in their entirety for any fungal segments expressing GFP. Autofluorescence within the range of GFP from substances such as minerals, organic debris, plant tissue, and other microbes was alleviated with a triple filter cube with DAPI/FITC/TRITC (excitation wavelengths of 380 400, 470 - 490 and 545 - $565 \mathrm{~nm}$; dichroic mirror wavelengths of 425 - 470, 500 - 540 and $570 \mathrm{~nm}$; and barrier wavelengths of 450 - 465, 510 - 540 and 580 - $620 \mathrm{~nm}$ ). From each object, two images were captured under both FITC and TRITC filters with a Hamamatsu digital camera (model C4742-95), and color-combined using MetaMorph computer software. A conversion factor from pixels (of captured images) to micrometers was determined as a length of $0.99 \mu \mathrm{m}$ using ImagePro Plus imaging software. For each captured image, radii and lengths of visible hyphae were measured using ImagePro Plus imaging software. Calculated biomass values were expressed as $\mu \mathrm{g} / \mathrm{g}$ of soil. Recoverable populations expressed as colony-forming units (CFU) per gram of soil were also determined by plating soil serial dilutions on selective medium containing hygromycin B.

\subsection{Statistical Analysis}

Experimental data were analysed using SAS package 9.0 (SAS Institute, Cary, NC, USA). Linear regression was performed for Ct values vs. log-transformed fungal DNA concentrations and for Trichoderma harzianum ThzID1-M3 mycelial weights vs. DNA estimates from the real-time PCR assay. Estimated amounts of ThzID1-M3 DNA and Sclerotinia sclerotiorum DNA in sclerotia were analyzed over time using analysis of variance with significant differences occurring at the level of $\mathrm{P}<0.05$. Estimated amounts of ThzID1-M3 DNA and Sclerotinia sclerotiorum DNA in sclerotia were analyzed over time using analysis of variance.

\section{Results and Discussion}

We previously observed that the presence of $A$. saprophilus nematodes both reduced radial growth of $T$. harzia- 
num ThzID1-M3 colonies, as well as recoverable ThzID1-M3 populations in soil [6], showing a deleterious effect of nematode predation on ability of this potential biocontrol fungus to survive and proliferate in the presence of the fungivorous nematodes. Evidence of this trophic effect was substantiated by the concurrent significant population increase of A. saprophilus in soil to which $T$. harzianum had been added [6]. A trophic cascade effect is implicated, evidenced by a significant reduction in colonization of sclerotia of $S$. sclerotiorum by $T$. harzianum, when the fungivorous nematodes were present [6].

qRT-PCR proved to be an effective tool for monitoring and quantifying changes in amounts of DNA of the respective fungal organisms in sclerotia, and of T. harzianum ThzID1-M3 in soil. The TMeGFP2 and TMSCL2 primer/probe sets amplified only their specific targets (ThzID1-M3 and Sclerotinia sclerotiorum DNA, respectively). In the real-time PCR assay with the TMeGFP2 set, no artificial signals were observed from sclerotia in soil or from soil where ThzID1-M3 was not applied (controls). DNA of ThzID1-M3 was detected inside sclerotia whenever the mycoparasite was applied to soil.

Results from qRT-PCR analysis of T. harzianum ThzID1-M3 DNA in soil showed that the total amount of DNA of the applied fungus in soil increased initially but then decreased significantly (Figure 2). Fungal biomass, as determined by epifluorescence microscopy, followed a similar pattern (Figure 2). There was a significant effect of sample time on biomass measurements determined by microscopy and DNA levels determined by qRT-PCR $(P<0.05)$. Mean estimates of ThzID1-M3 DNA peaked after approximately 3 days. Fluorescent hyphal segments were undetectable at the end of the experimental period; however the fungus could still be recovered on isolation plates, suggesting that it was primarily present as conidia in soil. Microscopy with image analysis quantified only active hyphae, whereas qRT-PCR presumably quantified all of ThzID1-M3 propagules including conidia; however the biomass of individual conidia is small relative to hyphal segments.

The amount of T. harzianum ThzID1-M3 DNA inside of colonized sclerotia generally followed a similar temporal pattern (Figure 3). However, amounts of ThzID1-M3 DNA were much higher in sclerotia: mean DNA mass per sclerotium was approximately 100-fold higher than per gram of soil (Figure 2 \& Figure 3), even though individual sclerotia weigh considerably less than one gram. As the mycoparasite $T$. harzianum proliferated inside sclerotia, levels of DNA of the host fungus (S. sclerotiorum) dropped significantly over time (Figure 3). These results support strongly support the hypothesis that sclerotia can serve as a refuge from predation by fungivorous nematodes as well as possibly from antagonistic effects of other soil microbes, allowing $T$. harzianum to persist and even proliferate away from its natural enemies. However, one alternative or concurrent explanation may be that the nutritional environment inside of sclerotia is more favorable for growth of the mycoparasite.

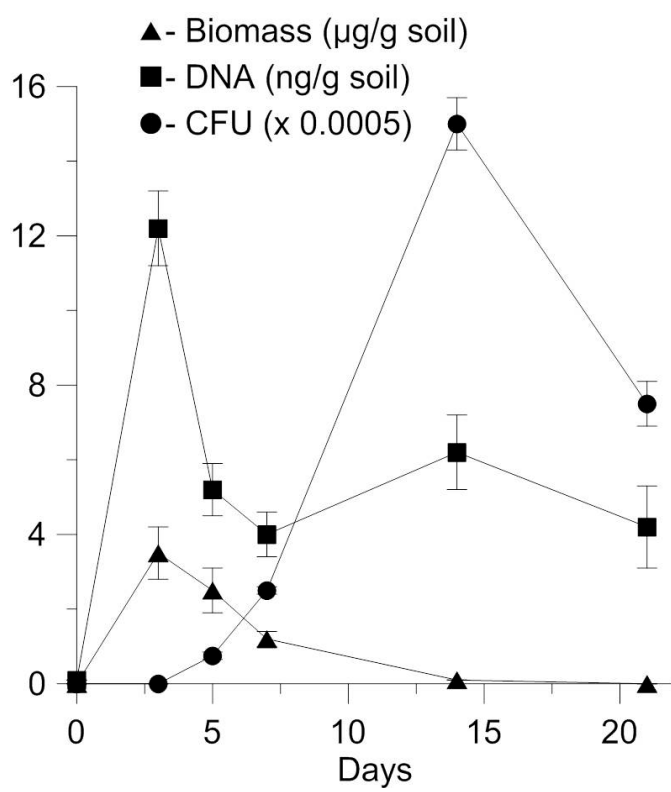

Figure 2. Biomass, DNA, and colony-forming units (CFU) of Trichoderma harzianum ThzID1-M3 recovered from soil, as measured by epifluorescence microscopy, qRT-PCR, and dilution plating, respectively. 


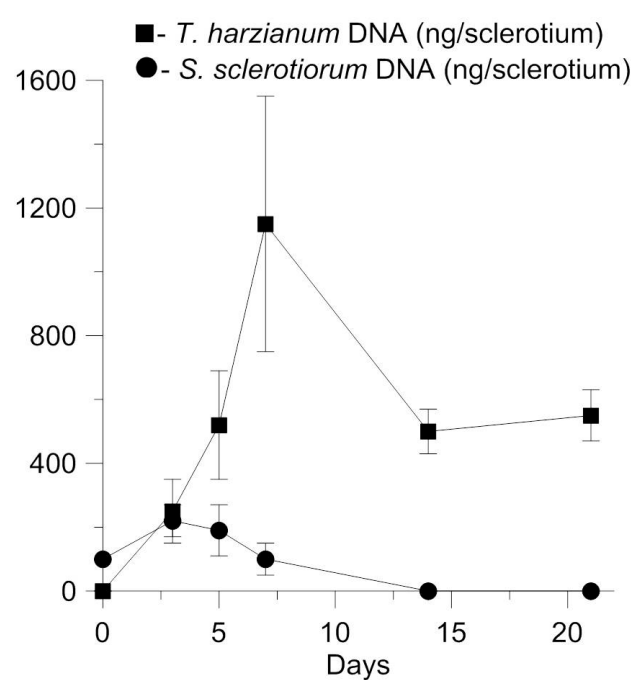

Figure 3. DNA of Trichoderma harzianum ThzID1-M3 and Sclerotinia sclerotiorum recovered from sclerotia, as measured by qRT-PCR.

Both accurate detection and quantification of mycoparasitism of sclerotia-forming pathogens by introduced biocontrol fungiare necessary for evaluation of biocontrol efficacy. It is possible that similar trophic dynamics may be involved for other mycoparasitic fungi that have been shown to infect and colonize sclerotia of plant fungi [12] [13]. Characterization of ecologically complex microbial systems is challenging, but a number of tools are available to help unravel this complexity. These tools include the use of molecular transformation to provide biocontrol agents with useful marker genes, epifluorescence and confocal laser scanning microscopy, and qRT-PCR to provide sensitive analysis of DNA levels in soil. Fierer et al. [14] noted that while high levels of bacterial and fungal diversity make quantifying and characterizing soil microbial community dynamics a challenging task, qRT-PCR has emerged as a promising tool for such analysis. For example, qRT-PCR effectively measured fungal DNA extracted from soil using different methods [15], and offers advantages over microscopic methods [16].

In this study, qRT-PCR effectively quantified colonization of sclerotia by the introduced mycoparasite $T$. harzianum ThzID1-M3, and allowed comparison with growth and persistence in the surrounding soil where natural enemies were present. qRT-PCR also allows quantification of the increase or decrease of host (sclerotium) DNA, unlike microscopy. Overall, these results support the hypothesis that sclerotia may provide a spatial refuge for mycoparasitic fungi from natural enemies such as the fungivorous nematode $A$. saprophilus. As described by Hawkins et al. [17], refuges "provide a general mechanism for interpreting ecological patterns at both the community level (their species diversity) and population level (their dynamics).” We anticipate that qRT-PCR will continue to be a useful tool for helping to unravel the ecological complexity of soil microbial systems.

\section{References}

[1] Harman, G.E., Petzoldt, R., Comis, A. and Chen, J. (2004) Interactions between Trichoderma harzianum Strain T22 and Maize Inbred Line Mo17 and Effects of These Interactions on Diseases Caused by Pythiuin ultimum and Colletotrichum graminicola. Phytopathology, 94, 147-153. http://dx.doi.org/10.1094/PHYTO.2004.94.2.147

[2] Vasas, V. and Jordan, F. (2006) Topological Keystone Species in Ecological Interaction Networks: Considering Link Quality and Nontrophic Effects. Ecological Modelling, 196, 365-378. http://dx.doi.org/10.1016/j.ecolmodel.2006.02.024

[3] Harman, G.E., Howell, C.R., Viterbo, A., Chet, I. and Lorito, M. (2004) Trichoderma Species-Opportunistic, Avirulent Plant symbionts. Nature Reviews Microbiology, 2, 43-56. http://dx.doi.org/10.1038/nrmicro797

[4] Knudsen, G.R., Eschen, D.J., Dandurand, L.M. and Bin, L. (1991) Potential for Control of Sclerotinia sclerotiorum through Colonization of Sclerotia by Trichoderma harzianum. Plant Disease, 75, 466-470.

[5] Knudsen, G.R., Eschen, D.J., Dandurand, L.M. and Wang, Z.G. (1991) Method to Enhance Growth and Sporulation of Pelletized Biocontrol Fungi. Applied and Environmental Microbiology, 57, 2864-2867. 
[6] Bae, Y.-S. and Knudsen, G.R. (2001) Influence of a Fungus-Feeding Nematode on Growth and Biocontrol Efficacy of Trichoderma harzianum. Phytopathology, 91, 301-306. http://dx.doi.org/10.1094/PHYTO.2001.91.3.301

[7] Wardle, D.A., Williamson, W.M., Yeates, G.W. and Bonner, K.I. (2005) Trickledown Effects of Aboveground Trophic Cascades on the Soil Food Web. Oikos, 111, 348-358. http://dx.doi.org/10.1111/j.0030-1299.2005.14092.x

[8] Pace, M.L., Cole, J.J., Carpenter, S.R. and Kitchell, J.F. (1999) Trophic Cascades Revealed in Diverse Ecosystems. Trends in Ecology \& Evolution, 14, 483-488. http://dx.doi.org/10.1016/S0169-5347(99)01723-1

[9] Bae, Y.S. and Knudsen, G.R. (2000) Cotransformation of Trichoderma harzianum with $\beta$-Glucuronidase and Green Fluorescent Protein Genes Provides a Useful Tool for Monitoring Fungal Growth and Activity in Natural Soils. Applied and Environmental Microbiology, 66, 810-815. http://dx.doi.org/10.1128/AEM.66.2.810-815.2000

[10] Kim, T.G. and Knudsen, G.R. (2008) Quantitative Real-Time PCR Effectively Detects and Quantifies Colonization of Sclerotia of Sclerotinia sclerotiorum by Trichoderma spp. Applied Soil Ecology, 40, 100-108. http://dx.doi.org/10.1016/j.apsoil.2008.03.013

[11] Orr, K.A. and Knudsen, G.R. (2004) Use of Green Fluorescent Protein and Image Analysis to Quantify Proliferation of Trichoderma harzianum in Nonsterile Soil. Phytopathology, 94, 1383-1389. http://dx.doi.org/10.1094/PHYTO.2004.94.12.1383

[12] Sarrocco, S., Mikkelsen, L., Vergara, M., Jensen, D.F., Lubeck, M. and Vannacci, G. (2006) Histopathological Studies of Sclerotia of Phytopathogenic Fungi Parasitized by a GFP Transformed Trichoderma virens Antagonistic Strain. Mycological Research, 110, 179-187. http://dx.doi.org/10.1016/j.mycres.2005.08.005

[13] Stewart, A. and Harrison, Y. (1988) Mycoparasitism of sclerotia of Sclerotium cepivorum. Australasian Plant Pathology, 18, 10-14. http://dx.doi.org/10.1071/APP9890010

[14] Fierer, N., Jackson, J.A., Vilgalys, R., and Jackson, R.B. (2005) Assessment of Soil Microbial Community Structure by Use of Taxon-Specific Quantitative PCR Assays. Applied and Environmental Microbiology, 71, 1429-1432. http://dx.doi.org/10.1128/AEM.71.7.4117-4120.2005

[15] Kabir, S., Rajendran, N., Amemiya, T. and Itoh, K. (2003) Quantitative Measurement of Fungal DNA Extracted by Three Different Methods Using Real-Time Polymerase Chain Reaction. Journal of Bioscience and Bioengineering, 96, 337-343. http://dx.doi.org/10.1016/S1389-1723(03)90133-2

[16] Kim, T.G. and Knudsen, G.R. (2011) Comparison of Real-Time PCR and Microscopy to Evaluate Sclerotial Colonisation by a Biocontrol Fungus. Fungal Biology, 115, 317-325. http://dx.doi.org/10.1016/j.funbio.2010.12.008

[17] Hawkins, B.A., Thomas, M.B. and Hochberg, M.E. (1993) Refuge Theory and Biological Control. Science, 262, 14291432. http://dx.doi.org/10.1126/science.262.5138.1429 